\title{
New extraction of color-octet NRQCD matrix elements from charmonium hadroproduction *
}

\author{
M. A. Sanchis-Lozano ${ }^{a, b \dagger}$ \\ (a) Departamento de Física Teórica \\ (b) Instituto de Física Corpuscular (IFIC) \\ Centro Mixto Universitat de València-CSIC \\ Dr. Moliner 50, E-46100 Burjassot, Valencia (Spain)
}

\begin{abstract}
We re-analyze Tavatron data on charmonium hadroproduction in the framework of the color-octet model implemented in the event generator PYTHIA taking into account initial-state radiation of gluons and AltarelliParisi evolution of final-state gluons fragmenting into $c \bar{c}$ pairs. We obtain new values for the color-octet matrix elements relevant to this production process. We discuss the sensitivity of our results to the transverse momentum lower cut-off employed in the generation to avoid the problematic $p_{t} \rightarrow 0$ region, arguing about the reliability of our previous extraction of the NRQCD matrix elements for the ${ }^{3} S_{1}^{(8)}$ and ${ }^{1} S_{0}^{(8)}+{ }^{3} P_{J}^{(8)}$ contributions. Finally we extrapolate to LHC energies to get predictions on the $J / \psi$ direct production rate.
\end{abstract}

\section{INTRODUCTION}

In a series of previous papers 1 - 4 we analyzed charmonium hadroproduction in the light of the color-octet model [5]. We employed a Monte Carlo generator (PYTHIA 5.7) [6] to assess the importance of some higher-order QCD effects. As a consequence we concluded that NRQCD matrix elements [7] determined from Tevatron experimental data $[8]$ were considerably overestimated in other analysis 91, since they absorb some perturbative effects, e.g. initial-state emission of gluons. The latter gives rise to an effective transverse momentum, enhancing the high- $p_{t}$ tail of the differential distribution of charmonium. Once taken into account, the extracted long-distance parameters have to be lowered significantly. This is especially apparent for the linear combination of matrix elements (MEs) ${ }^{1} S_{0}^{(8)}+{ }^{3} P_{0}^{(8)}$ where, in fact, a large discrepancy w.r.t. HERA results on $J / \psi$ photoproduction has been pointed out [10].

However, in our former work we did not consider Altarelli-Parisi (AP) evolution of the fragmenting gluon into charmonium for the ${ }^{3} S_{1}^{(8)}$ con-

\footnotetext{
${ }^{*}$ Work partially supported by CICYT under contract AEN-96/1718

†E-mail: mas@evalo1.ific.uv.es
}

tribution at high $p_{t}$. In this paper we implement such an effect benefitting from the evolution performed by PYTHIA itself as will be explained below. We shall show that although our previous numerical values for the ${ }^{3} S_{1}^{(8)} \mathrm{ME}$ were somewhat low, no significant change occurs for the corresponding ${ }^{1} S_{0}^{(8)}+{ }^{3} P_{0}^{(8)}$ ME. We also discuss the sensitivity to the lower cut-off used to avoid the singular $p_{t} \rightarrow 0$ region. We get started with the latter point in the following section.

\section{SENSITIVITY TO THE $p_{t}^{\min }$ CUT- OFF}

Production cross sections which are singular at $p_{t}=0$ are automatically regularized in running PYTHIA by setting a $p_{t}$ lower cut-off at $1 \mathrm{GeV}$ [6]. Consistently with our aim of using by-default options whenever possible in the generation, we kept this value in all our previous analysis [1, 他.

In fact, the differential cross section can have a singular behaviour at low $p_{t}$ even to next-toleading order due to imperfect cancellation of real and virtual contributions. However, from a NLO calculation of charmonium production by Mangano [11], one may conclude that our previously used lower cut-off $\left(p_{t}^{\text {min }}=1 \mathrm{GeV}\right)$ is con- 
sistent with the threshold obtained by Mangano ( $\approx 1 \mathrm{GeV}$ for the ${ }^{1} S_{0}^{(8)}$ channel), such that the averaged cross section is zero below this value.

Nevertheless, in order to assess the significance of the cut-off on the values of the long-distance parameters we performed new fits to Tevatron data by changing $p_{t}^{\text {min }}$. We found that, by varying $p_{t}^{\text {min }}$ in the $[1,2] \mathrm{GeV}$ range, the ${ }^{3} S_{1}^{(8)} \mathrm{ME}$ showed almost no appreciable change, whereas the ${ }^{1} S_{0}^{(8)}+{ }^{3} P_{0}^{(8)} \mathrm{ME}$ changed by a factor two at most [12].

Therefore, we keep in our subsequent analysis the value $p_{t}^{\text {min }}=1 \mathrm{GeV}$ as a self-consistent lower cut-off.

\section{ALTARELLI-PARISI EVOLUTION}

At high transverse momentum, gluon fragmentation via the color octet mechanism becomes the dominant source of charmonium production. On the other hand, AP evolution of the splitting gluon produces a depletion of its energy which has to be taken into account. If not so, the longdistance parameter for the ${ }^{3} S_{1}^{(8)}$ channel would be underestimated.

In this work the AP evolution of the fragmenting gluon was carried out from the evolution of the gluonic partner of the $J / \psi$ in the final state of the production channel

$g g \rightarrow g^{*}(\rightarrow J / \psi) g$

the technical reason being that the splitting gluon $g^{*}$ actually is not generated in our code [3]. (Notice however that the MEs used in the generation include the long-distance evolution of the $(c \bar{c})$ bound state [3].)

Indeed, on the average the fragmenting gluon should evolve in the transverse plane similarly to the other final-state gluon in (1), once corrected its virtuality to become of the order of the charmonium mass.

Thereby we get a correcting factor to be applied event by event to the transverse momentum of the generated $J / \psi$ (for the ${ }^{3} S_{1}^{(8)}$ channel only):

$x_{p}=\frac{\sqrt{P_{t}^{* 2}+m_{J / \psi}^{2}}}{\sqrt{P_{t}^{2}+m_{J / \psi}^{2}}}$ where $P_{t}\left(P_{t}^{*}\right)$ is the transverse momentum of the final-state gluon without (with) AP evolution.

At high $p_{t}$,

$p_{t}^{A P}=x_{p} \times p_{t}$

where $p_{t}$ is the transverse momentum of the $J / \psi$ as generated by PYTHIA (i.e. without AP evolution).

Although the above way to implement AP evolution may be somewhat indirect, it remains in the spirit of our whole analysis, i.e. using PYTHIA algorithms whenever possible. In fact it provides an energy depletion of the fragmenting gluon in accordance with Cho and Leibovich's work 9.

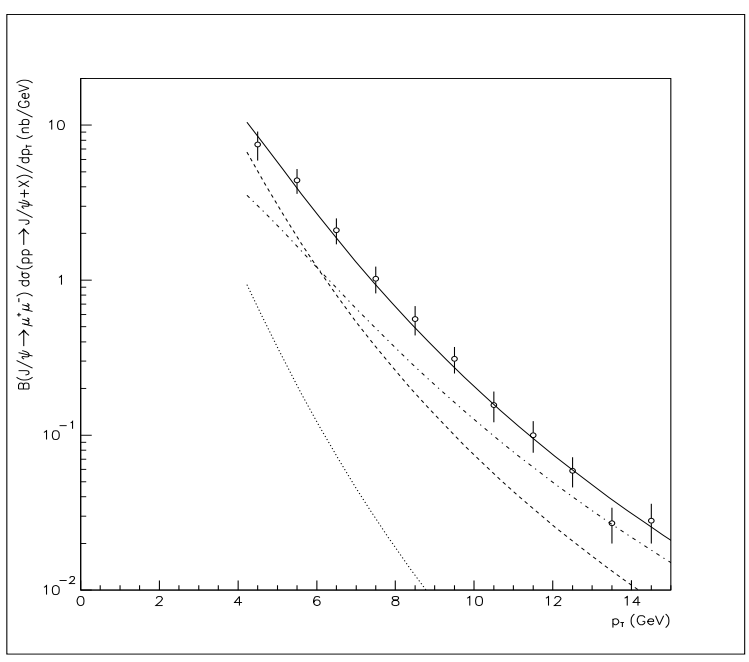

Figure 1. Curves obtained from PYTHIA for $J / \psi$ production at the Tevatron, including AP evolution and initial-state radiation on for the CTEQ $2 \mathrm{~L}$ parton distribution function. The charm mass was set equal to $1.48 \mathrm{GeV}$. (i) dotted line: Colorsinglet model, (ii) dashed line: ${ }^{1} S_{0}^{(8)}+{ }^{3} P_{0}^{(8)}$, (iii) dot-dashed line: ${ }^{3} S_{1}^{(8)}$, (iv) solid line: all contributions. 


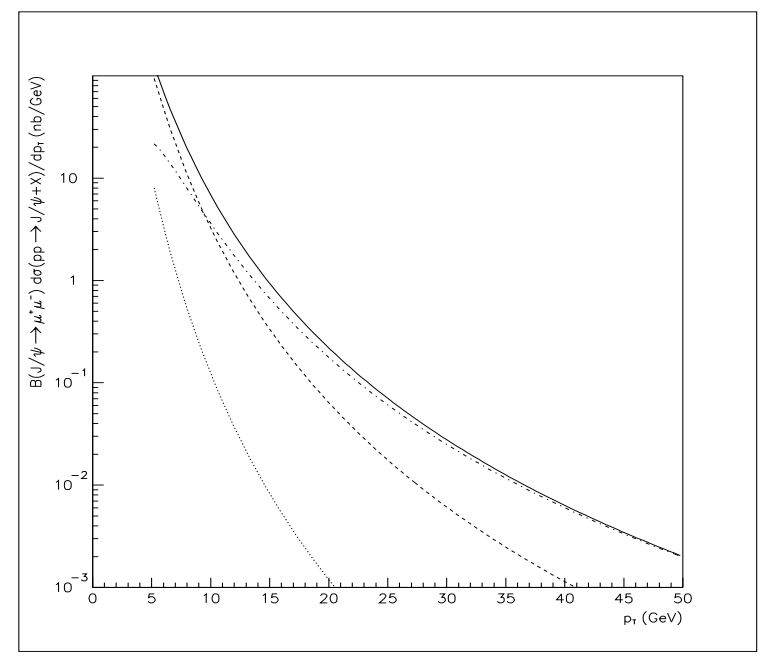

Figure 2. Curves obtained from PYTHIA for $J / \psi$ production at the LHC, without AP evolution and initial-state radiation on for the CTEQ $2 \mathrm{~L}$ parton distribution function. (i) dotted line: Color-singlet model, (ii) dashed line: ${ }^{1} S_{0}^{(8)}+{ }^{3}$ $P_{0}^{(8)}$, (iii) dot-dashed line: ${ }^{3} S_{1}^{(8)}$, (iv) solid line: all contributions.

\section{NEW EXTRACTION OF COLOR- OCTET MES}

We have performed new fits to the Tevatron data [8] using three PDFs (as in [3]) but now with AP evolution implemented in the generation as described in the preceding section. In table 1 we show the numerical values for $\left\langle O_{8}\left({ }^{3} S_{1}\right)\right\rangle$ and the linear combination $M_{3}=3 \times\left(\frac{\left\langle\mathrm{O}_{8}\left({ }^{3} P_{0}\right)>\right.}{m_{c}^{2}}+\right.$ $\left.\frac{\left\langle O_{8}\left({ }^{1} S_{0}\right)>\right.}{3}\right)$ obtained in our analysis. The new values for the $<O_{8}\left({ }^{3} S_{1}\right)>$ matrix elements have been increased by a factor about 3 w.r.t. AP off [3]. Conversely the $M_{3}$ value is slightly decreased, moreover remaining quite smaller than in other analysis [9]. Therefore we conclude that the $M_{3}$ value obtained in our work is reliable and in a better agreement with HERA results on $J / \psi$ photoproduction 13.

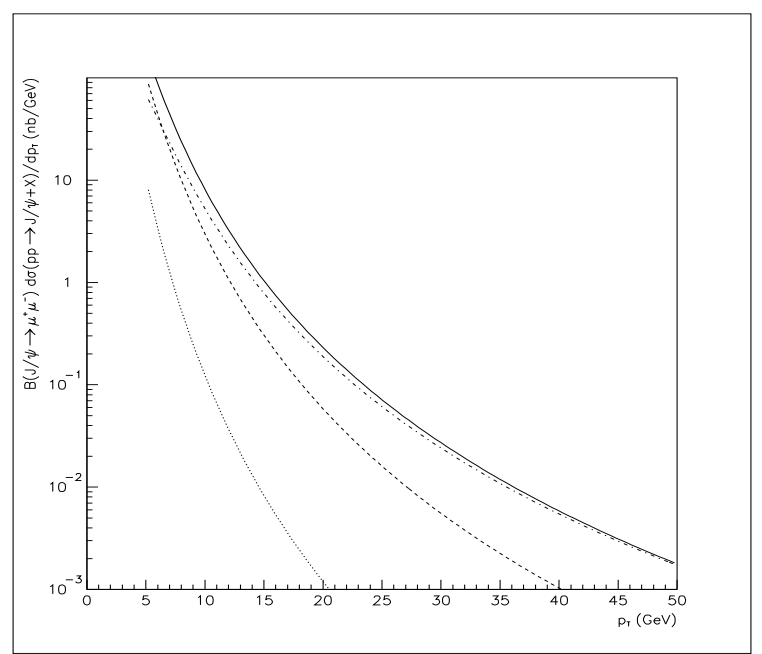

Figure 3. Curves obtained from PYTHIA for $J / \psi$ production at the $\mathrm{LHC}$, including AP evolution and initial-state radiation on for the CTEQ $2 \mathrm{~L}$ parton distribution function. (i) dotted line: Color-singlet model, (ii) dashed line: ${ }^{1} S_{0}^{(8)}+{ }^{3}$ $P_{0}^{(8)}$, (iii) dot-dashed line: ${ }^{3} S_{1}^{(8)}$, (iv) solid line: all contributions.

\section{5. $J / \psi$ DIRECT PRODUCTION RATE AT THE LHC}

In order to get an estimate of the expected charmonium production rate at the LHC, we ran PYTHIA with the color-octet model implemented in. In figure 3 we show the predicted differential cross section times the muonic branching fraction of $J / \psi$ with AP evolution included. In figure 2 we show the same curve with AP off. By comparison, one may conclude that, once adjusted the longdistance parameters to fit Tevatron data, there is no significant difference between both generations.

Let us stress that because of theoretical uncertainties associated to different choices for the charm mass, energy scales, parton distribution function ..., the theoretical curves of figures 2 and 3 have to be considered as order-of-magnitude 
Table 1

Color-octet matrix elements (in units of $10^{-3} \mathrm{GeV}^{3}$ ) from the best fit to Tevatron data on direct $J / \psi$ production for different PDFs. Initial-state radiation of gluons and AP evolution as explained in the text were swichted on. For comparison we quote the values given by Cho and Leibovich: (6.6 \pm 2.1$)$ and $(66 \pm 15)$ respectively.

\begin{tabular}{lcc}
\hline ME: & $\left\langle O_{8}\left({ }^{3} S_{1}\right)>\right.$ & $M_{3}=3 \times\left(\frac{\left\langle O_{8}\left({ }^{3} P_{0}\right)\right\rangle}{m_{c}^{2}}+\frac{\left\langle O_{8}\left({ }^{1} S_{0}\right)\right\rangle}{3}\right)$ \\
\hline CTEQ2L & $9.6 \pm 1.5$ & $13.2 \pm 2.1$ \\
MRSD0 & $6.8 \pm 1.6$ & $13.2 \pm 2.1$ \\
GRVHO & $9.2 \pm 1.1$ & $4.5 \pm 0.9$ \\
\hline
\end{tabular}

predictions. Nevertheless the relatively high production rate at high- $p_{t}$ (of the order of the $\mathrm{pb}$ at $p_{t}=50 \mathrm{GeV}$ ) makes especially interesting the analysis of charmonia production at the LHC. A preliminary study on $\Upsilon(1 S)$ prompt production leads to a similar conclusion for the bottomonia family.

\section{CONCLUSIONS}

After checking our analysis by varying the lower $p_{t}$ cut-off set in the Monte Carlo generation to avoid the problematic $p_{t} \rightarrow 0$ region, we conclude that our former choice $p_{t}^{\text {min }}=1 \mathrm{GeV}$ was justified and our study of charmonium hadroproduction trustworthy. On the other hand, once implemented Altarelli-Parisi evolution in our framework, the new extracted values of the color-octet ME $<O_{8}\left({ }^{3} S_{1}\right)>$ increase (table 1$)$, recovering a similar result as in previous extractions [9]. On the other hand, the numerical values obtained for $M_{3}$ even decrease slightly, reinforcing the conclusions reported in our former work 114 .

With respect to the extrapolation of the $J / \psi$ production rate up to LHC energy, the theoretical prediction incorporating AP evolution (figure 3) does not differ significantly from the curve obtained without AP evolution (figure 2). This means that, from a practical point of view, there is no need to modify the code for a fast generation as shown in [3] if the corresponding coloroctet matrix elements are duly employed. Finally we conclude that the analysis of charmonia (and bottomonia) prompt production at the LHC deserves special attention in its own right.

\section{Acknowledgments}

I thank the LHC Workshop b-production subgroup for interesting discussions and suggestions.

\section{REFERENCES}

1. M.A. Sanchis-Lozano and B. Cano-Coloma, Nucl. Phys. B (Proc. Suppl.) 55A (1997) 277.

2. B. Cano-Coloma and M.A. Sanchis-Lozano, Phys. Lett. B 406 (1997) 232.

3. B. Cano-Coloma and M.A. Sanchis-Lozano, Nucl. Phys. B 508 (1997) 753.

4. M.A. Sanchis-Lozano, Nucl. Phys. B (Proc. Suppl.) 75B (1999) 191.

5. E. Braaten and S. Fleming, Phys. Rev. Lett. 74 (1995) 3327.

6. T. Sjöstrand, Comp. Phys. Comm. 82 (1994) 74.

7. G.T. Bodwin, E. Braaten, G.P. Lepage, Phys. Rev. D 51 (1995) 1125.

8. CDF Collaboration, F. Abe at al., Phys. Rev. Lett. 69 (1992) 3704; 71 (1993) 2537; 75 (1995) $1451 ; 79$ (1997) 578.

9. P. Cho and A.K. Leibovich, Phys. Rev. D 53 (1996) 6203.

10. M. Cacciari and M. Krämer, Phys. Rev. Lett. 76 (1996) 4128.

11. M. Mangano, private communication.

12. M.A. Sanchis-Lozano, 2nd meeting of the bproduction working group, LHC99 Workshop, April 1999.

13. B.A. Kniehl and G. Kramer, Eur. Phys. J. C 6 (1999) 493. 